\title{
NORMAL CONGRUENCE SUBGROUPS OF THE $t \times t$ MODULAR GROUP 1
}

\author{
BY M. NEWMAN
}

Communicated by A. M. Gleason, June 5, 1963

Let $\Gamma$ denote the group of rational integral $t \times t$ matrices of determinant 1 . If $n$ is a positive integer, $\Gamma(n)$ denotes the principal congruence subgroup of $\Gamma$ of level $n$, consisting of all elements of $\Gamma$ congruent modulo $n$ to a scalar matrix. The subgroup of $\Gamma(n)$ consisting of all elements of $\Gamma$ congruent modulo $n$ to the identity matrix is denoted by $\Gamma_{1}(n)$. Then $\Gamma(n), \Gamma_{1}(n)$ are normal subgroups of $\Gamma$. A subgroup $G$ of $\Gamma$ containing a principal congruence subgroup $\Gamma(n)$ is termed a congruence subgroup, and is said to be of level $n$ if $n$ is the least such integer. Notice that $\Gamma_{1}(n)$ is not in general a congruence subgroup, according to the definition above.

Let $p$ be a prime. Let $\mathrm{SL}(t, p)$ denote the group of $t \times t$ matrices with elements from $\mathrm{GF}(p)$ and determinant 1 , and let $H(t, p)$ denote the normal subgroup of $\operatorname{SL}(t, p)$ consisting of all scalar matrices. Then

$$
\mathrm{SL}(t, p) \cong \Gamma / \Gamma_{1}(p), \quad H(t, p) \cong \Gamma(p) / \Gamma_{1}(p),
$$

and $\operatorname{SL}(t, p), H(t, p)$ are of orders

$$
p^{t^{2}-1} \prod_{j=2}^{t}\left(1-p^{-j}\right),(t, p-1)
$$

respectively. In his book on the linear groups [1] Dickson proves that for $t>2, H(t, p)$ is a maximal normal subgroup of $\operatorname{SL}(t, p)$ and this of course implies that $\Gamma(p)$ is a maximal normal subgroup of $\Gamma$. This result is used to prove the theorem that follows:

THEOREM 1. Suppose that $t>2$. Then every normal congruence subgroup of odd level of $\Gamma$ is a principal congruence subgroup.

The theorem is also true for $t=2$, if the level is prime to 6 . (The case $t=2$ for the group of linear fractional transformations has been treated in [3].) Since the structure of the proof of Theorem 1 is identical with that of the proof for $t=2$ given in [3], we only indicate the points of difference, and refer the reader to [3] for full details. The proof is arranged for an induction and what is actually proved is the slightly more general theorem that follows:

1 The preparation of this note was supported by the Office of Naval Research. 
THEOREM 2. Suppose that $t>2$. Let $m, n$ be positive integers, $m$ odd. Let $G$ be a normal subgroup of $\Gamma$ such that $\Gamma(n) \supset G \supset \Gamma(m n)$. Then $G=\Gamma(n d), d \mid m$.

In order to prove this theorem generally it is necessary to give special proofs for the cases when $m$ is a prime or the square of a prime. If $m$ is any prime and $(m, n)=1$ then the theorem of Dickson referred to above implies the result. If $m$ is an odd prime and $m \mid n$, then $\Gamma(n) / \Gamma(m n)$ is abelian of type $(m, m, \cdots, m)$ and it is not difficult to show that the normality of $G$ implies that $G=\Gamma(n)$ or $\Gamma(m n)$. If $m$ is the square of an odd prime, then the proofs given in [3] go over unchanged, with one exception: the commutator subgroup $\Gamma^{\prime}$ of $\Gamma$ is no longer of index 6 in $\Gamma$ (as is the case for $t=2$ and $\Gamma$ the group of linear fractional transformations) but is in fact just $\Gamma$ itself. This has been proved by Hua and Reiner (see [2]), although some care must be taken in interpreting their results since they consider the more general unimodular group in which the determinant is allowed to be -1 as well. The formal structure of the induction remains unchanged.

\section{REFERENCES}

1. L. E. Dickson, Linear groups, Dover, New York, 1958.

2. L. K. Hua and I. Reiner, Automorphisms of the unimodular group, Trans. Amer. Math. Soc. 71 (1951), 331-348.

3. M. Newman, Normal congruence subgroups of the modular group, Amer. J. Math. (to appear).

National Bureau of Standards 